
\title{
QCD THERMODYNAMICS WITH LIGHT QUARKS AND GLUEBALL SPECTRA WITH DYNAMICAL
QUARKS*t
}

D. K. SINCLAIR

HEP Division, Argonne National Laboratory, 9700 S. Cass Ave., Argonne, II.,60439

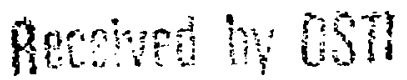

MAY O 91990
The submited manugrigt has been authore by a contractor of the U.S. Govemmen under contract No. W-31-109ENG-3E Actordinglv, the U.S. Government tetains honexelusive. tovalty-tree license to oublis or reptorduce the published form comiribution, of allow others :- . . U.S. Government purposes.

Simulations of Lattice QCD with 4 flavours of light staggered quarks $(m=.025)$ were performed on a $12^{3} \times 4$ lattice, confirming the first order nature of the transition. Lattice QCD with a light isodoublet of staggered quarks $(m=.0125)$, and a heavier singlet $(m=.25)$ was studied, also on a $12^{3} \times 4$ lattice. The order of the transition was less clear. Improved glueball wavefunctions have been used to study glueball spectra in theories incorporating dynamical quarks.

\section{INTRODUCTION}

For intermediate quark masses ( $m \gtrsim .1$ for 4 flavours, $m \gtrsim .025$ for 2 flavours), the first order phase transitions reported on smaller lattices $\left(8^{3} \times 4\right.$ and smaller) appeared to be finite size effects when simulations were performed on larger lattices $\left(10^{3} \times 1\right.$ and larger) ${ }^{1}$. We therefore felt it necessary to reexamine the smaller mass region to determine whether the first order transitions observed there ${ }^{1,2}$ were finite volume effects. We have therefore extended our 4 flavour simulations at $m=.025$ to a $12^{3} \times 4$ lattice ${ }^{3}$ and those with 2 flavours ( $u$ and $d$ ) with $m=.0125$ and 1 (s) with $m=.25$, also to a $12^{3} \times 4$ lattice ${ }^{4}$. This reports work done in collaboration with J. B. Kogut.

Using improved glueball wave functions, TeperMichael $^{6}$ and the APE collaboration ${ }^{6}$ have managed to make reliable predictions of the glueball masses in pure gauge theories. Of considerable interest is what happens when dynamical quarks are included and glueball meson mixing can occur. As a preliminary investigation of these effects we have studied the glueball spectra and topological charge in SU(2) theories with 4 dynamical quarks $(m=.05-.1)$ on both a $10^{3} \times 20$ and a $6^{3} \times 16$ lattice ${ }^{7}$. This reports work done in collaboration with J. B. Kogut and $\mathbf{M}$. J. Teper. ${ }^{\ddagger}$ In addition we are studying these quantities in the more realistic SU(3) theories on a $12^{4}$ lattice at $m=.01$ and $m=.025$, using the configurations produced by the HEMCGC "Grand Challenge" collaboration. This reports work being done as part of the HEMCGC collaboration. $\S$

Section 2 reports the 4 flavour thermodynamics, section 3 the $2+1$ flavour thermodynamics, while section 4 gives preliminary results of the glueball mass/topological charge calculations.

\section{QCD THERMODYNAMICS WITH 4 LIGHT FLAVOURS 3 \\ QCD with 4 light staggered quarks was simu-} lated on a $12^{3} \times 4$ lattice using the hybrid method. Runs of up to 140,000 sweeps (1400 time units) were made at various values of $\beta=6 / g^{2}$ close to the chiral phiase transition. Separate runs made from hot and cold starts at $\beta=4.98$ showed clear evidence for coexisting states, strong evidence for a first order phase transition at $\beta=4.98$ (1) (Fig. 1).

*Talk presented at the LATTICE '89 Conference, Capri, Italy, September 18-21, 1989.

tWork supported by the U.S. Department of Energy, Division of High Energy Physics, Contract W-31-109ENG-38.

\# thank M. J. Teper and J. B. Kogut for allowing me to present this preliminary data prior to its submission for publication.

§์K. M. Bitar, A. D. Kennedy, W. Liu, T. A. Degrand, S. Gottlieb. J. B. Kogut, R. L. Renken, M. C. Ogilvie, P. Rossi, D. K. Sinclair, R. L. Sugar, M. J. Teper, D. Toussaint. 


\section{$\beta=4.98$}

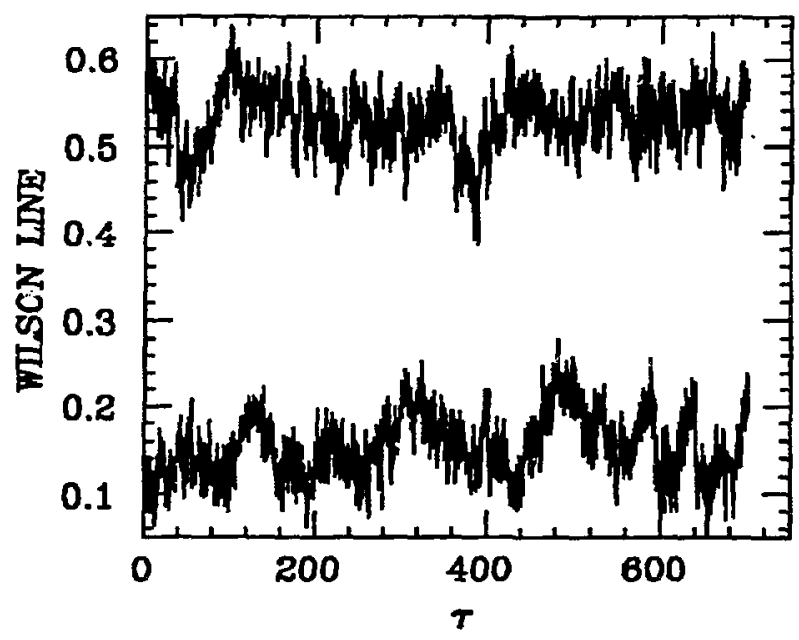

FIGURE 1

Time evolution of the Wilson Line from hot and cold starts at $\beta=4.98$ on a $12^{3} \times 4$ lattice.

The discontinuities of various order parameters across the transition on the $12^{3} \times 4$ lattice as compared with those on the $8^{3} \times 4$ lattice are

$$
\begin{array}{rll}
\delta\langle\bar{\psi} \psi\rangle= & 12^{3} \times 4 & 8^{3} \times 4 \\
\delta W L=3(13) & .6(1) \\
\delta P \operatorname{la} q= & .373(9) & .35(5) \\
& .0372(9) & .06(1)
\end{array}
$$

This good agreement strengthens the conclusion that we are indeed seeing a true first order transition as distinct from a finite volume effect.

Finally we have measured the energy densities at each $\beta$. The discontinuities in each of the gluon and fermion energy densities at the transition, corrected for finite size effects are,

$$
\begin{aligned}
& \delta \epsilon_{g} / T^{4}=13.6(5) \\
& \delta \epsilon_{f} / T^{4}=9.7(3)
\end{aligned}
$$

to zeroth order in $g^{2}$. The inclusion of the higher order (in $g^{2}$ ) terms as well as the calculation of the pressure are reported in a forthcoming paper.
These runs were performed on the Argonne HEP Division's ST-100 array processor.

\section{QCD WITH A REALISTIC QUARK SPECTRUM ${ }^{4}$ \\ QCD with a light isodoublet of quarks ( $u$ and $d$ )} of mass $m=.0125$ and a heavier isosinglet quark $(\mathrm{s})$ of mass $m=.25$ was simulated on a $12^{3} \times 4$ lattice using the hybrid method with "noisy" fermions to enable us to tune the number of quark ilavours. Runs of typically 30,000 sweeps (300 time units) were made for several $\beta$ values in the vicinity of the finite temperature transition, at $\beta=5.197(3)$. Although the transition was clearly abrupt, clear sigrals of metastability were absent. There were, however, abrupt jumps in the time evolution of order parameters close to the transition, suggestive of the tunnelings expected at a first order transition (Figs. 2,3). It would require considerably more statistics to determ;ne if this represents true metastability. What was clear was that the width of the metastability region, if we are seeing signs of metastability, was considerably narrower than for the $S^{3} \times 4$ lattice.

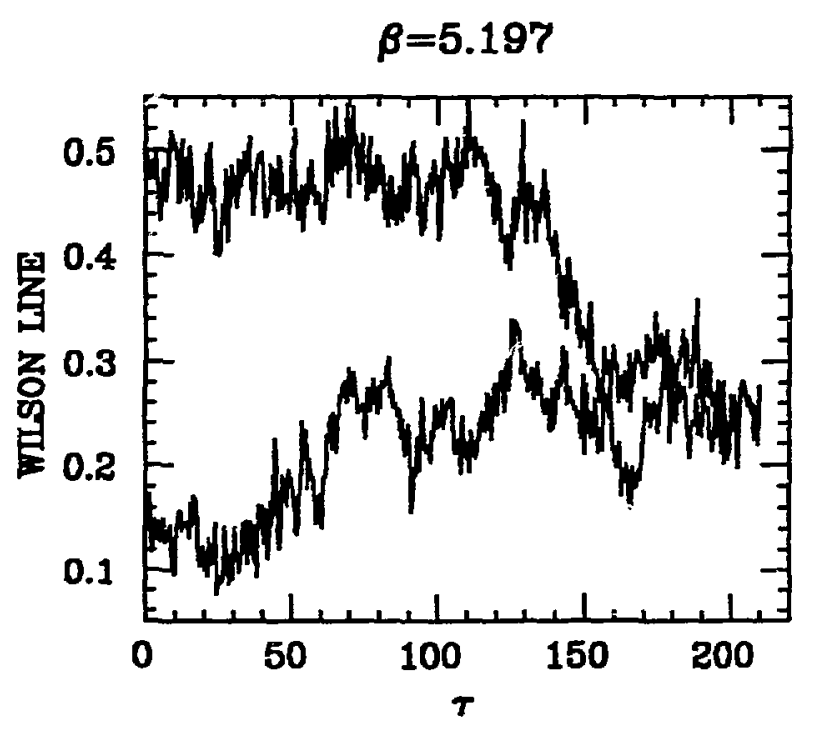

FIGURE 2

Time evolution of the Wilson Line at $\beta=5.197$. 


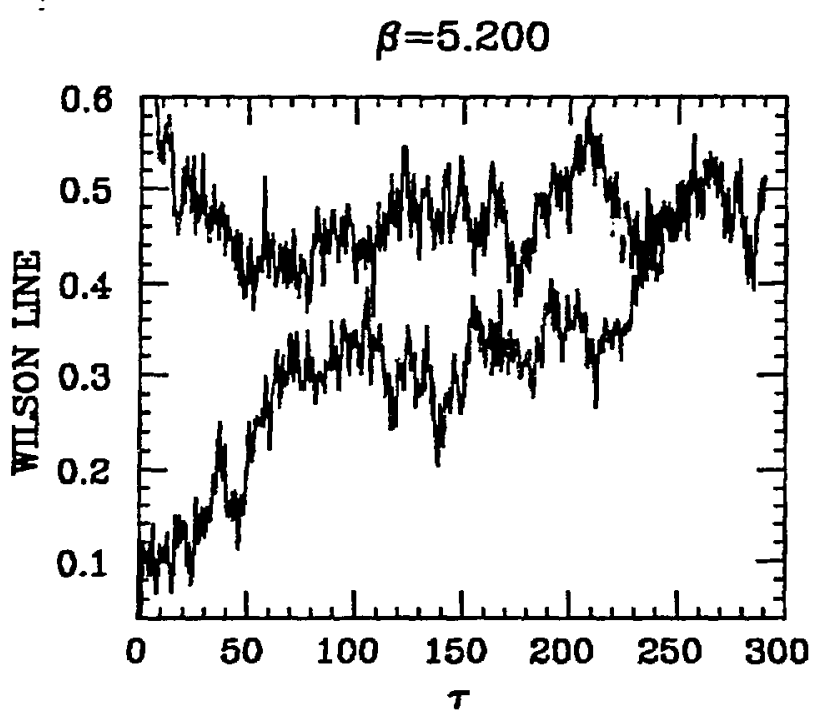

FIGURE 3

Time evolution of the Wilson Line at $\beta=5.200$.

We have measured the energy densities in the neighbourhood of this transition and find (to zeroth order in $g^{2}$ ) the following discontinuities in the energy densities of the gluons, up + down and strange quarks, at the transition.

$$
\begin{array}{rcc} 
& 12^{3} \times 4 & 8^{3} \times 4 \\
\delta \epsilon_{g} / T^{4}= & 10.80(100) & 12.00(200) \\
\delta \epsilon_{u, d} / T^{4}= & 4.77(25) & 6.30(100) \\
\delta \epsilon_{s} / T^{4}= & 1.73(10) & 1.70(25)
\end{array}
$$

This good agreement indicates that the finite volume effects are small. These numbers indicate considerable $s \bar{s}$ production when hadronic matter is heated beyond this transition.

These simulations were performed on the CRAY $Y-M P$ at PSC and the CRAY $2 S$ at NCSA.

\section{GLUEBALLS AND TOPOLOGICAL CHARGE WITH DYNAMICAL QUARKS}

We have measured the glueball masses in SU(2) lattice gauge theory with four flavours of dynamical quarks as a preliminary study of the effect of the inclusion of dynamical quarks on the glueball spectrum ${ }^{\vec{T}}$. This is important because the possibility of gluebaii-meson mixing and glueball decay into mesons is likely to change the glueball spectrum, and could suppress glueballs completely.
We use the method of Teper-Michael to construct improved ("Fuzzy") glueball wave functions. For the quenched theory these give a dramatic improvement in the signal/noise ratio and permit accurate measurement of the glueball and toron masses by measuring glueball propagators at relatively small separations. It appears that they give similar improvement with dynamical quarks.

Preliminary results from $20010^{3} \times 20$ lattices at $\beta=1.975$ (confined) and quark masses $m=.1$ and $m=.05$ yield the following mass ratios (extrapolated to $m=0$ ).

$$
\begin{aligned}
M(2+) / M(0+) & =1.4(1) \\
M(0-) / M(2+) & =1.2(2) \\
M(0+) / M(\rho) & =1.1(3) \\
\sqrt{(\sigma)} / M(\rho) & =.27(3)
\end{aligned}
$$

Topological charge was measured for each of the above lattices using the cooling method ${ }^{9}$ to remove short distance lattice artifacts, the value of the topological charge $Q$ being

$$
Q=1 / 32 \pi^{2} \sum F \tilde{F}
$$

for the cooled quark lattice. In addition this quantity was measured for $6^{3} \times 16$ lattices at the same $\beta$ (deconfined).

One expects that the topological susceptibility will have the behaviour

$$
\begin{aligned}
& \left\langle Q^{2}\right\rangle \sim m \quad \text { (confined) } \\
& \left\langle Q^{2}\right\rangle \sim m_{f}^{n=4} \quad \text { (deconfined) }
\end{aligned}
$$

Preliminary results are

$$
\begin{aligned}
\left\langle Q^{2}\right\rangle \sim m^{1.37(21)} & \text { (confined) } \\
\left\langle Q^{2}\right\rangle \sim m^{5.9(11)} & \text { (deconfined } m=.1-.075) \\
\left\langle Q^{2}\right\rangle \sim m^{3.6(8)} & \text { (deconfined } m=.075-.05 \text { ) }
\end{aligned}
$$

in good agreement with the theoretical expectations.

This work was performed on the CRAY Y-MP at PSC.

For the SU(3) case work is in progress by the HEMCGC to analyse the 500 gauge configurations at $m=.01$ and 500 with $m=.025$ each produced on a $12^{4}$ lattice with 2 flavours of staggered quarks at $\beta=5.6$ calculating both glueball propagators and topological charge for each configuration. 
Although the analysis was too embryonic to yield even preliminary results for this meeting, it appeared that, for $m=.01$ the masses of the $0^{++}$and $2^{++}$ states were around 1 with the $2^{++}$heavier than the $0^{++}$. This compares with the $\rho$ mass of $.55(1)$ reported at this meeting 10 .

This analysis is being performed on the ETA-10G at SCRI as part of the HEMCGC Grand Challenge project.

\section{REFERENCES}

1. M. Fukugita and A. Ukawa, Phys. Rev. Lett. 57, 507 (1987); J. B. Kogut and D. K. Sinclair, Nucl. Phys. B280[FS18], 625 (1987); R. Gavai, Nucl. Phys. B269, 530 (1986); R. Gupta, G. Guralnik, G. W. Kilcup, A. Patel, and S. R. Sharpe, Phys. Rev. D38, 1288 (1988); Kh. Bitar, A. D. Kennedy, R. Horsley, S. Meyer and P. Rossi, Nucl. Phys. B313, 348 (1989); J. B. Kogut and D. K. Sinclair, Phys. Rev. D39, 636 (1989); S. Gottlieb, W. Liu, R. L. Renken, R. L. Sugar and D. Toussaint, Nucl. Phys. B (Proc. Suppl.) 9, 326 (1989); R. Gupta, Nucl. Phys. B (Proc. Suppl.) 9, 473 (1989).

2. R. Gavai, J. Potvin and S. Sanlielevici, Phys. Rev. Lett. 58, 251 (1987); F. Karsch, J. B. Kogut, D. K. Sinclair and H. W. Wyld, Phys. Lett. B188, 353 (1987); S. Gottlieb, W. Liu, D. Toussaint, R. L. Renken and R. L. Sugar, Phys. Rev. Lett. 59, 1513 (1987) and Phys. Rev. D35, 3972 (1987); J. B. Kogut, E.V.E. Kovacs and
D. K. Sinclair, Nucl. Phys. B290[FS20], 431 (1987); J. B. Kogut, and D. K. Sinclais, Phys. Rev. Lett. 59, 399 (1987); A. Ukawa, talk presented at this conference (1989).

3. D. K. Sinclair and J. B. Kogut, Phys. Lett. B229, 93 (1989).

4. J. B. Kogut and D. K. Sinclair, Phys. Lett. B229, 107 (1989).

5. M. Teper, Phys. Lett. B183, 345 (1987); C. Michael, M. Teper, Phys. Lett. B199, 95 (1987) and Nucl. Phys. B305, 453 (1988); C. Michael, G. Tickle and M. Teper, Phys. Lett. B207, 313 (1988); C. Michael and M. Teper, Phys. Lett. B206, 299 (1988) and Nuci. Phys. B314, 347 (1989).

6. The Ape collaboration: M. Albanese et al., Phys. Lett. B192, 163 (1987) and Phys. Lett. B197, 400 (1987).

7. J. B. Kogut, M. J. Teper and D. K. Sinclair (in preparation).

8. J. B. Kogut and D. K. Sinclair (in preparation).

9. M. Teper, Phys. Lett. B162, 357 (1985) and Phys. Lett. B171, 81 (1986); J. Hoek, M. Teper and J. Waterhouse, Phys. Lett. B180, 112 (1986) and Nucl. Phys. B288, 589 (1987); M. Teper, Phys. Lett. B202, 553 (1988).

10. W. Liu, talk presented at this conference (1989).

\section{DISCLAIMER}

\footnotetext{
This report was prepared as an account of work sponsored by an agency of the United States Government. Neither the United States Government nor any agency thereof, nor any of their employees, makes any warranty, express or implied, or assumes any legal liability or responsibility for the accuracy, completeness, or usefulness of any information, apparatus, product, or process disclosed, or represents that its use would not infringe privately owned rights. Reference herein to any specific commercial product, process, or service by trade name, trademark, manufacturer, or otherwise does not necessarily constitute or imply its endorsement, recommendation, or favoring by the United States Government or any agency thereof: The views and opinions of authors expressed herein do not necessarily state or reflect those of the United States Government or any agency thereof.
} 